\title{
Selectable one-step PCR-mediated integration of a degron for rapid depletion of endogenous human proteins
}

\author{
Ryan M. Sheridan and David L. Bentley \\ Department of Biochemistry and Molecular Genetics, University of Colorado School of Medicine, Aurora, CO
}

BioTechniques 60:69-74 (February 2016) doi 10.2144/000114378

Keywords: genome editing; CRISPR, homology directed repair; protein destabilization domain; regulated protein stability; human degron mutants

Supplementary material for this article is available at www.BioTechniques.com/article/114378.

Manipulation of protein stability with ligand-regulated degron fusions is a powerful method for investigating gene function. We developed a selectable cassette for easy C-terminal tagging of endogenous human proteins with the E. coli dihydrofolate reductase (eDHFR) degron using CRISPR/Cas9 genome editing. This cassette permits high-efficiency recovery of correct integration events using an in-frame self-cleaving $2 \mathrm{~A}$ peptide and the puromycin resistance gene. PCR amplified donor eDHFR cassette fragments with 100 bases of homology on each end are integrated by homology-directed repair (HDR) of guide RNA (gRNA)-targeted double-stranded DNA breaks at the 3' ends of open reading frames (ORFs). As proof of principle, we generated cell lines in which three endogenous proteins were tagged with the eDHFR degron. When the antibiotic trimethoprim is removed from the media, each of the eDHFR-tagged proteins was depleted by $>90 \%$ within 2-4 h, and this depletion was reversed by re-addition of trimethoprim. Since puromycin selection permits recovery of in-frame degron fusions with high efficiency using only 100-bp long regions of homology, this method should be applicable on a genome-wide scale for generating libraries of conditional mutant cell lines.

The ability to knock out genes by CRISPR/ Cas9 mediated genome editing is revolutionizing modern genetics. Libraries of human knockout cell lines generated by error-prone non-homologous end joining (NHEJ) are valuable tools that open up new ways of screening for novel phenotypes and drug sensitivities (1-5). Engineered knockout lines have the advantage over short hairpin RNA (shRNA) knockdown in that off-target effects are minimized and complete rather than partial protein depletion is achieved (6-8). The value of gene knockouts is generally limited to non-essential genes, however, and conditional mutant technologies are required to extend the scope of high-throughput functional genomics to essential genes. Conditionally expressed shRNAs (9) are useful for this purpose, but are limited by off-target effects (10), partial depletion, and slow kinetics particularly for stable proteins $(11,12)$.

Conditional protein depletion that is completely specific for the target protein can be achieved by fusion to a degron domain that, under appropriate conditions, targets the tagged protein for proteasomal degradation $(13,14)$. The first portable degron, introduced by Varshavsky and colleagues (15), was a temperature-sensitive variant of mammalian dihydrofolate reductase (DHFR) that when fused to a target protein can induce rapid degradation at $37^{\circ}$ in yeast. The DHFR degron is stabilized against unfolding and degradation by binding to its ligand, methotrexate (15). Other liganddependent degrons have been adapted for use in mammalian cells. Rapid degra- dation of exogenously expressed proteins has been achieved in mammalian cells with the auxin-dependent degradation system co-opted from plants $(16,17)$. However, this system requires modification of the host cells to express the plant TIR1 E3 ubiquitin ligase.

Other degrons confer constitutive instability unless a stabilizing ligand is present. These degrons have the advantage that they do not require the ectopic expression of any additional factors. A mutant variant of FK506 binding protein (FKBP) functions as a degron that is antagonized by binding to the cell-permeable ligand, Shield-1 (18). The FKBP-based degron has been used in conjunction with CRISPR/Cas9 genome editing with donor plasmids to N-terminally tag several endogenous human proteins

\section{METHOD SUMMARY}

We describe a PCR-based method for one-step C-terminal tagging of endogenous human proteins with the E. coli dihydrofolate reductase degron using CRISPR/Cas9 genome editing. The cassette used for protein tagging contains a self-cleaving 2A peptide-puromycin resistance gene fusion for efficient selection of in-frame integration events using only 100 -bp long regions of homology. This method makes it feasible to generate genome-wide libraries of conditional mutant cell lines. 
$(19,20)$. These studies demonstrate the feasibility of the degron approach for endogenous human proteins, but the depletion achieved was relatively slow. For example, removal of the Shield-1 ligand induced $50 \%$ degradation of TCOF1 over 6 h (19). These previously used approaches required construction of plasmid donors, so they are not suitable for high-throughput applications.

The E. coli DHFR (eDHFR) degron developed by Wandless and colleagues permits extensive depletion of exogenously expressed proteins in mammalian cells and C. elegans $(21,22)$. The eDHFR degron is stabilized by tight binding to the antibiotic trimethoprim (TMP), which is innocuous in eukaryotic cells (23). Proteins tagged with eDHFR are constitutively degraded unless the cells are exposed to TMP (22). The level of tagged protein can be directly controlled by modulating the TMP concentration in the growth medium (22). Unlike shRNA methods this degron-based strategy is advantageous since depletion kinetics are not limited by the natural protein half-life, which allows for more rapid knockdown of stable proteins. The eDHFR/TMP system has not previously been tested for depletion of endogenous human proteins.

Here we describe a cassette for selectable C-terminal tagging of endogenous human proteins with the eDHFR degron using CRISPR/Cas9 genome editing. PCR amplified donor fragments derived from this cassette were integrated by homology-directed repair (HDR) of DNA breaks made close to the 3 ' ends of open reading frames (ORFs). High-frequency recovery of correct integration events was achieved using an in-frame self-cleaving $2 \mathrm{~A}$ sequence fused to the puromycin resistance gene. Each of three degron fusions engineered by this strategy were rapidly depleted in a TMP-dependent way. This one-step method requires PCR products with only 100 bp of homology at each end and is therefore suitable for genome-wide construction of conditional mutant libraries.

\section{Materials and methods}

\section{Plasmids}

The pX330 plasmid (24) was obtained from Addgene (Cambridge, MA) (plasmid \#42230). NELF-A, Xrn2, and Dis3 specific guide RNAs (gRNAs) were selected using the gRNA design tool provided by the Zhang lab (http:// crispr.mit.edu/). Each gRNA was inserted into pX330 as described by Ran et al. (25).
pAc5 HA3-eDHFR-T2A-puro was derived from pAc5gRNA Cas9 (26) by replacing the EcoRl-Hindlll fragment encoding Cas9 with a PCR fragment encoding HA3-eDHFR amplified from pBMN DHFR(DD)-YFP (22) (Addgene plasmid \#29325) with primers HA3-eDHFRfw and eDHFRrev (see Supplementary Material). This degron has R12Y, G67S, and Y100I mutations in eDHFR that were introduced by Iwamoto et al. to optimize degron function (22). The Y100I mutation inhibits enzymatic activity (27). pAc5 HA3-eDHFR-T2A-neo was generated from pAc5 HA3-eDHFR-T2A-puro by replacing the Nhel-Xhol fragment encoding the puro' gene with a PCR fragment encoding the neo ${ }^{R}$ gene derived from pMini Tol2 SV40 Neo (28).

\section{Transfections and colony selection} All experiments were performed using HAP1 myeloid leukemia cells (29) grown in IMDM (GE Healthcare Bio-Sciences, Pittsburgh, PA) supplemented with 10\% FBS, 4 mM L-glutamine, and 1\% penicillin/streptomycin. PCR primers used to generate the linear HDR donors are listed in the Supplementary Material, and the genomic regions used for designing the homology arms are shown in the Supplementary Material. Two phosphorothioate linkages were included at the $5^{\prime}$ end of each HDR PCR primer. HAP1 cells were seeded into $10 \mathrm{~cm}$ dishes at $70 \%$ confluency. Prior to transfection, $10 \mu \mathrm{M}$ trimethoprim was added from a $10 \mathrm{mM}$ stock in DMSO. HAP1 cells were co-transfected with $5 \mu \mathrm{g}$ each of $\mathrm{pX} 330 \mathrm{gRNA}$ plasmid and linear HDR donor using FuGENE HD (Promega, Madison, WI) or Lipofectamine 2000 (Thermo Fisher Scientific, Waltham, MA). Puromycin (750 ng/mL) was added $72 \mathrm{~h}$ after transfection, and the medium was changed every 2-3 days. After 1-2 weeks, colonies were transferred to 24-well plates, and genomic DNA was isolated for PCR genotyping.

\section{PCR genotyping}

Primers and primer binding sites are described in the Supplementary Material. For the NELFA-eDHFR colonies, NELFA_ degron-F/NELFA_degron-R (322 bp product) were used to screen for the presence of the wild-type allele, and NELFA_degron-F/ DHFR_degron_HA-R (308 bp product) were used to screen for incorporation of the HA-eDHFR-T2A-puro ${ }^{\mathrm{R}}$ cassette. For the Xrn2-eDHFR colonies, Xrn2_degron-F/ Xrn2_degron-R (371 bp product) were used to screen for the presence of the wild-type allele, and Xrn2_degron-F/DHFR_degron_
HA-R (270 bp product) were used to screen for incorporation of the HA-eDHFRT2A-puro ${ }^{R}$ cassette. For the Dis3-eDHFR colonies, Dis3_Cterm-F/Dis3_Cterm-R (460 bp product) were used to screen for the presence of the wild-type allele, and Dis3_Cterm-F/DHFR_degron_HA-R (301 bp product) were used to screen for incorporation of the HA-eDHFR-T2A-puro ${ }^{R}$ cassette. To verify that the cassette was correctly incorporated at the 3' end of each target gene, the eDHFR specific PCR products for NELFAeDHFR colonies 1, 2, and 5, Xrn2-eDHFR colonies 3 and 5, and Dis3-eDHFR colonies 2, 3, and 4 were sequenced using the DHFR degron_HA-R primer.

\section{Western blot analysis}

Cells were either harvested (+TMP control) or washed $2 \times$ with $5 \mathrm{mLPBS}$ and fresh medium was added containing 0.1\% DMSO (-TMP). For the recovery experiment, after $24 \mathrm{~h}$, the -TMP medium was replaced with medium containing $10 \mu \mathrm{M}$ TMP (T7883; SigmaAldrich, St. Louis, MO) for the indicated time points. Protein was isolated with M-PER reagent (Thermo Fisher Scientific) containing $1 \mu \mathrm{g} / \mathrm{mL}$ pepstatin, $1 \mathrm{mM}$ benzamidine, 0.2 $\mathrm{mM}$ sodium orthovanadate, and $1 \mathrm{mM}$ PMSF, quantified by Bradford assay, and electrophoresed on Bio-Rad (Hercules, CA) Criterion 10\% Tris-HCl gels. Gels were transferred to PVDF membranes (IPVH00010; Millipore, Billerica, MA) and blocked in 5\% milk/PBS-Tween overnight at $4^{\circ} \mathrm{C}$, then incubated for $2 \mathrm{~h}$ at room temperature with anti-HA (1:1000) (12CA5; Roche, Basel, Switzerland), anti-NELF-A (1:250) (sc-23599; Santa Cruz Biotechnology, Dallas, TX), or rabbit anti-CstF77 (30). A Bio-Rad ChemiDoc MP imaging system was used to quantify each band. The background values for each lane were subtracted from the corresponding band signals. Each band signal was normalized by dividing by the backgroundsubtracted CstF77 loading control signal. The values were expressed relative to the +TMP control sample for each blot.

\section{Results and discussion}

A selectable cassette for high efficiency C-terminal tagging with the eDHFR degron

To make C-terminal fusions of the eDHFR degron in-frame with natural target genes, we constructed a 1521 bp cassette with a single ORF comprising a 3×Gly linker, 3xHA-tag, eDHFR degron, Thoseaasigna virus T2A 
A

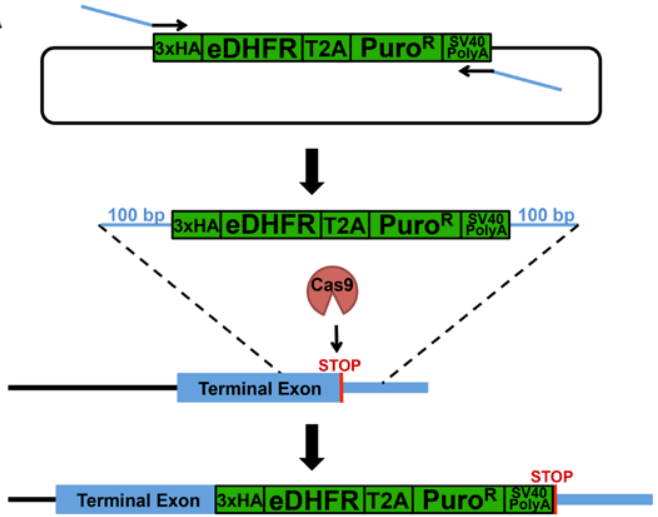

1. Generate linear HDR donors using PCR

2. Co-transfect HDR donor and pX330 at a 1:1 ratio

$\downarrow 72$ hours

3. Begin selection in puromycin

$\downarrow 1-2$ weeks

4. Isolate and expand single colonies

5. PCR Genotyping

B

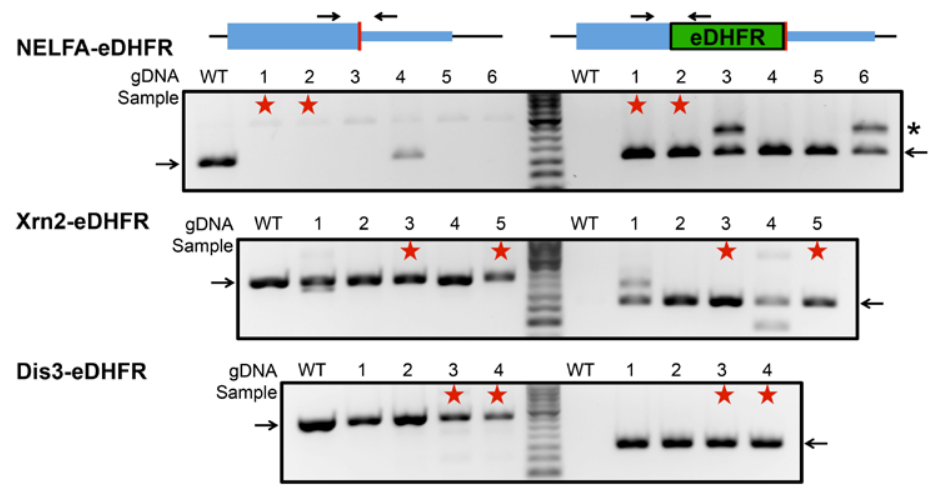

Figure 1. Efficient integration of eDHFR degron tag using PCR products with short homology arms. (A) Workflow for insertion of a C-terminal 3XHA-eDHFR tag using CRISPR/Cas9-mediated homology directed repair (HDR) to integrate a PCR-amplified cassette with 100-bp homology arms. The cassette contains a selectable $P$ uro $^{R}$ gene and self-cleaving T2A sequence that are in-frame with the target ORF. (B) PCR was used to genotype each Puro ${ }^{R}$ colony. Reactions were performed using primer sets specific for either the wild-type locus or the predicted insertion event. Diagrams above each set of reactions indicate the relative positions of the primer pairs. The colony number is listed above each lane. Arrows indicate the expected products. Colonies that were used for further experiments are indicated with a red star. Wildtype (WT) HAP1 genomic DNA was used for control reactions. * denotes unexpected PCR products.

self-cleaving peptide, and the puromycin resistance gene followed by an SV4O poly(A) site (Figure 1A). We PCR-amplified the HA-eDHFR-T2A-puro ${ }^{R}$ cassette to generate linear double-stranded DNA donors for HDR. The HDR donors contained homology arms added by PCR amplification with primers that had 100-nucleotide overhangs homologous to either side of the Cas9 cleavage site positioned close to the termination codon. Each donor was designed so that the HA-eDHFR-T2A-puro ${ }^{\text {R }}$ cassette would be incorporated in-frame after the last codon. C-terminal tagging has two potential advantages over the $\mathrm{N}$-terminal tagging strategies used previously. First, a large insertion at the termination codon in the last exon is less likely to disrupt splicing than an insertion at the initiation codon in one of the first exons, and secondly, C-terminal tagging is amenable to insertion of bar codes at an innocuous position downstream of the inserted SV40 poly(A) site at the $3^{\prime}$ end of the cassette. To test this scheme, we used CRISPR/Cas9 to insert the cassette at the $\mathrm{C}$-termini of three genes coding for the transcription elongation factor NELF-A and the exoRNases Xrn2 and Dis3 in three separate experiments. A single gRNA was designed for each gene that directs cleavage within 15 bases on either side of the termination codon. gRNA sequences were cloned into the pX330 plasmid (24) to allow for expression of the chimeric gRNA-tracrRNA along with the Streptococcus pyogenes Cas9 endonuclease. We co-transfected the donor PCR fragments with corresponding pX330 gRNA plasmids into HAP1 human myeloid leukemia cells (29) that were then maintained in medium supplemented with TMP $(10 \mu \mathrm{M})$. HAP1 cells can be maintained with a predominantly haploid genome; however, DNA content analysis revealed that our cultures contained mostly diploid cells.
After 1-2 weeks of selection, puromycinresistant colonies were picked.

The T2A self-cleaving peptide positioned between the eDHFR degron and the puro ${ }^{\mathrm{B}}$ protein (Figure $1 \mathrm{~A}$ ) allows the tagged target protein and the selectable marker to be expressed from the same transcript under the control of the endogenous target gene promoter. In principle, cells will only be puromycin-resistant if they accurately integrate the HA-eDHFR-T2Apuro $^{R}$ cassette. To test this prediction, we genotyped several puromycin-resistant colonies derived from each of the transfections targeting NELF-A, Xrn2 and Dis3. Two PCRs were performed for each clone using a common forward primer that sits upstream of the predicted integration site and a reverse primer specific for the wild-type allele or the HA-eDHFR-T2A-puro ${ }^{R}$ cassette (Figure 1B). For NELF-A, we screened six colonies, all of which produced a positive band for the eDHFR-specific reaction (Figure 1B, right panel). Colonies 1, 2, and 5 also lacked bands for the wild-type allele, suggesting that all copies of the NELF-A gene had incorporated the eDHFR tag (Figure 1B). We sequenced the PCR products for these colonies and verified that the eDHFR tag was integrated in-frame at the 3 end of the NELF-A gene in all three.

The five Xrn2-eDHFR colonies that we screened by PCR also showed incorporation of the eDHFR tag in all cases (Figure $1 \mathrm{~B}$, right panel). However, these five colonies also produced PCR products specific for the wild-type locus, indicating that the HA-eDHFR-T2A-puro ${ }^{R}$ cassette was only integrated at one allele. We sequenced the PCR products for colonies 3 and 5 and verified that the eDHFR degron was inserted in-frame at the $3^{\prime}$ end of the Xrn2 ORF in both cases. Similarly all four puromycin resistant Dis3-eDHFR colonies tested were PCR positive for insertion of the cassette but only at one allele. We sequenced the PCR products for Dis3-eDHFR colonies 2, 3 , and 4 and verified in-frame integration in all three cases. In summary, 15/15 puromycin resistant colonies tested in these experiments targeting 3 different genes had the degron cassette integrated at the $3^{\prime}$ end of the ORF, and in $8 / 8$ cases where the PCR product was sequenced, correct in-frame integration was confirmed. We conclude that selection for puromycin resistance dependent on in-frame insertion of the T2A-puro ${ }^{R}$ sequence is highly effective for isolating clones with correctly integrated 
C-terminal tags. For more efficient tagging of both alleles in a diploid cell, co-transfection with two donor PCR fragments bearing different selectable markers would be advantageous. For this purpose, we constructed an isogenic HA-eDHFR-T2A-neo ${ }^{R}$ cassette that is compatible with the same primers used to amplify the puro ${ }^{R}$ cassette. Recent advances in HDR technology could further increase the efficiency of donor incorporation using compounds that inhibit competing NHEJ $(31,32)$.

The power of the selection strategy used here is that it becomes possible to easily isolate correct integration products using donor PCR fragments with homology arms of only $100 \mathrm{bp}$. This method therefore requires significantly less effort than designing a separate donor plasmid with long regions of homology for each desired integration site $(19,20,33)$. Instead, our PCR-based approach allows for multiple HDR donors to be rapidly created using the same plasmid template and is therefore suitable for high-throughput construction of degron libraries. Furthermore, PCR generation of the HDR donors makes it possible to easily incorporate bar codes at the $3^{\prime}$ ends of the inserted cassettes to aid screening of libraries of degron mutant cell lines.

Regulated degradation of endogenous eDHFR-tagged human proteins

To assess the stability of tagged endogenous fusion proteins, we selected two independent colonies for each eDHFRtagged protein (NELF-A, Xrn2, and Dis3) and conducted Western blotting before and after washing out TMP for $24 \mathrm{~h}$ (Figure 2A). In each case protein levels were reduced to nearly undetectable levels. Quantitation relative to a control protein, CstF77, showed that NELFA-eDHFR and Dis3-eDHFR were depleted by over 95\% and Xrn2-eDHFR by $85 \%$. The latter estimate is a minimum value due to weak Western blot signals for HA-tagged Xrn2-eDHFR. There was good agreement between the levels of depletion in independent clones. We conclude that the eDHFR degron can effectively deplete endogenous human proteins to nearly undetectable levels.

To test the kinetics of NELFA-eDHFR and Dis3-eDHFR degradation, we washed TMP out for various times and collected protein samples for Western blotting (Figure 2, B and C). Compared with parental HAP1 cells, the NELFA-eDHFR line showed a higher molecular weight NELF-A immunoreactive

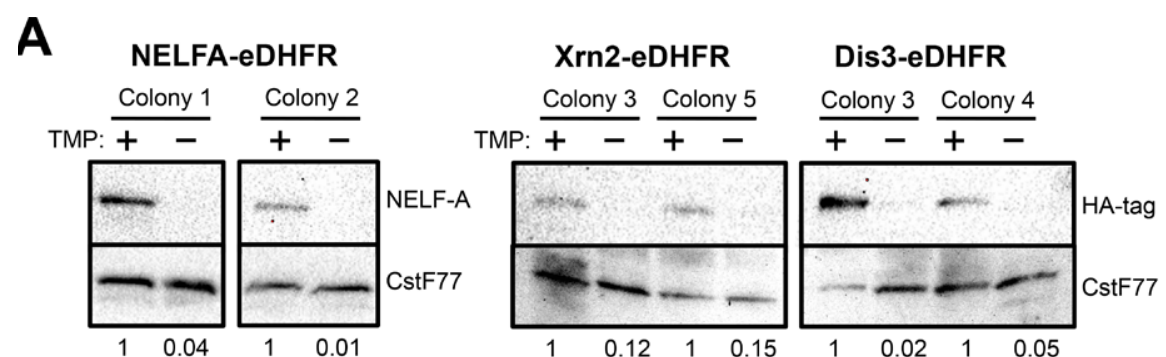

\section{B NELFA-eDHFR Colony 1}

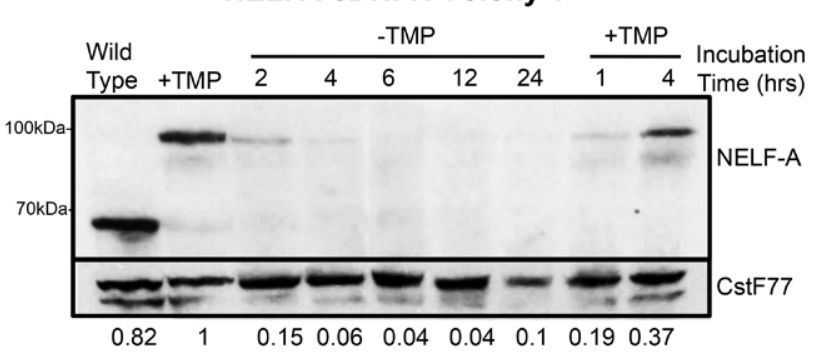

C

Dis3-eDHFR Colony 4

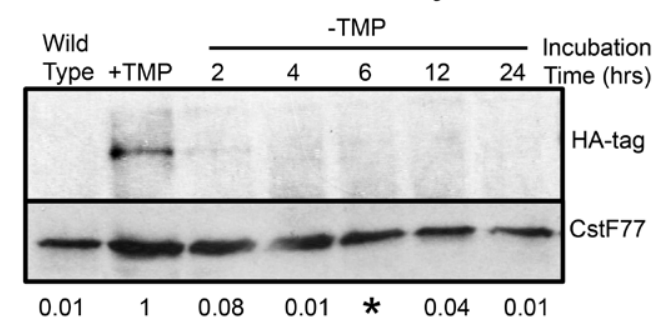

Figure 2. Rapid and reversible depletion of endogenous human proteins tagged with the eDHFR degron. (A) Western blots of eDHFR-tagged NELF-A, Xrn2, and Dis3. Cells were grown in $10 \mu \mathrm{M}$ TMP and then in -TMP media for an additional $24 \mathrm{~h}$. Bands were quantified relative to the CstF77 loading control and normalized to the +TMP lysate. (B) Time course of protein depletion and recovery for NELFAeDHFR. Cells grown in $10 \mu \mathrm{M}$ TMP were either harvested or washed and incubated in -TMP media for the indicated time points. After $24 \mathrm{~h}$, TMP $(10 \mu \mathrm{M})$ was added back for the indicated times. AntiNELF-A Western blots were quantified as described in (A). (C) Dis3-eDHFR depletion was assayed as described in (B) by anti-HA Western blotting. *Indicates that the signal was below background levels.

polypeptide corresponding to the fusion protein and no detectable wild-type NELF-A, consistent with all copies of the gene being edited (Figure 2B). In addition, we did not observe any noticeable reduction in NELFAeDHFR levels compared with wild-type NELF-A, indicating that the degron was fully stable in the presence of TMP. When TMP was withdrawn, NELFA-eDHFR was depleted by $85 \%$ within $2 \mathrm{~h}$. NELFA-eDHFR levels reached their lowest point of $4 \%-6 \%$ after 4-6 h (Figure 2B). We observed similar results for Dis3-eDHFR, which was depleted by over 95\% within $4 \mathrm{~h}$ (Figure 2C). The rate of depletion of these 2 eDHFR-tagged proteins is faster than that reported for the TCOF1-FKBP degron, which was reduced by $50 \%$ in $6 \mathrm{~h}$ (19).

A potential advantage of degrons over shRNAs is their reversibility. To test whether the depletion of endogenous eDHFR-tagged factors was reversible, NELFA-eDHFR cells were grown for $24 \mathrm{~h}$ in medium lacking TMP to fully deplete the protein and then TMP was added back. Recovery of NELFA-eDHFR protein was detectable within $1 \mathrm{~h}$ and further increased after $4 \mathrm{~h}$ (Figure 2B). We conclude that the eDHFR degron can achieve relatively rapid, complete, and reversible depletion of endogenous proteins under TMP control. However, additional work is required to determine whether these results apply broadly to most human proteins including those that are especially long-lived (12).

Here we presented a straightforward one-step method for generating human cell lines expressing endogenous eDHFRtagged proteins. We found that CRISPR/ Cas9-mediated HDR permits the efficient integration of our PCR-amplified HA-eDHFR- 
T2A-puro ${ }^{R}$ cassette ( 1.5 kb) using homology arms only 100-bp long. As proof of principle, we generated multiple independent human lines expressing 3 different eDHFR-tagged proteins and showed that withdrawal of TMP can effectively deplete a tagged protein within 4-6 h. Unlike shRNA methods, degronmediated depletion is not limited by the target protein's natural half-life, thereby permitting more rapid removal of target proteins. By combining the eDHFR degron with other ligand-dependent degrons $(16,18)$, one could independently control the depletion of multiple endogenous proteins in different combinations. The generation of HDR donors by PCR and selection of in-frame integration events using the T2A-puro ${ }^{R}$ gene greatly streamlines isolation of tagged cell lines, making our approach suitable for highthroughput generation of bar-coded eDHFR degron libraries suitable for screening of essential and non-essential genes.

\section{Authors contributions}

Both authors contributed to planning the experiments. R.S. carried out the experiments. Both authors contributed to writing the manuscript and approved the final manuscript.

\section{Acknowledgments}

This work was supported by $\mathrm{NIH}$ grants GM063873 and GM058613 to D.B. R.S. was supported by NIH-T32-GM008730. We thank S. Pradhan for help with cloning and M. Johnston and J. Degregori for helpful discussions. This paper is subject to the NIH Public Access Policy.

\section{Competing interests}

The authors declare no competing interests.

\section{References}

1. Shalem, O., N.E. Sanjana, E. Hartenian, X. Shi, D.A. Scott, T.S. Mikkelsen, D. Heckl, B.L. Ebert, et al. 2014. Genome-scale CRISPRCas9 knockout screening in human cells. Science 343:84-87.

2. Shalem, O., N.E. Sanjana, and F. Zhang. 2015. High-throughput functional genomics using CRISPR-Cas9. Nat. Rev. Genet. 16:299-311.

3. Koike-Yusa, H., Y. Li, E.P. Tan, C. VelascoHerrera Mdel, and K. Yusa. 2014. Genome-wide recessive genetic screening in mammalian cells with a lentiviral CRISPR-guide RNA library. Nat. Biotechnol. 32:267-273.

4. Parnas, O., M. Jovanovic, T.M. Eisenhaure, R.H. Herbst, A. Dixit, C.J. Ye, D. Przybylski, R.J. Platt, et al. 2015. A Genome-wide CRISPR
Screen in Primary Immune Cells to Dissect Regulatory Networks. Cell 162:675-686.

5. Chen, S., N.E. Sanjana, K. Zheng, O. Shalem, K. Lee, X. Shi, D.A. Scott, J. Song, et al. 2015. Genome-wide CRISPR screen in a mouse model of tumor growth and metastasis. Cell 160:1246-1260.

6. Baek, S.T., G. Kerjan, S.L. Bielas, J.E. Lee, A.G. Fenstermaker, G. Novarino, and J.G. Gleeson. 2014. Off-target effect of doublecortin family shRNA on neuronal migration associated with endogenous microRNA dysregulation. Neuron 82:1255-1262.

7. Singh, S., A.S. Narang, and R.I. Mahato. 2011. Subcellular fate and off-target effects of siRNA, shRNA, and miRNA. Pharm. Res. 28:2996-3015.

8. Song, H.W., A. Bettegowda, D. Oliver, W. Yan, M.H. Phan, D.G. de Rooij, M.A. Corbett, and M.F. Wilkinson. 2015. shRNA off-target effects in vivo: impaired endogenous siRNA expression and spermatogenic defects. PLoS ONE 10:e0118549.

9. Meerbrey, K.L., G. Hu, J.D. Kessler, K. Roarty, M.Z. Li, J.E. Fang, J.I. Herschkowitz, A.E. Burrows, et al. 2011. The pINDUCER lentiviral toolkit for inducible RNA interference in vitro and in vivo Proc Natl Acad Sci U S A. 108:3665-3670.

10. Marine, S., A. Bahl, M. Ferrer, and E. Buehler. 2012. Common seed analysis to identify off-target effects in siRNA screens. J. Biomol. Screen. 17:370378.

11. Schwanhäusser, B., D. Busse, N. Li, G. Dittmar, J. Schuchhardt, J. Wolf, W. Chen, and M. Selbach. 2011. Global quantification of mammalian gene expression control. Nature 473:337-342.

12. Toyama, B.H., J.N. Savas, S.K. Park, M.S. Harris, N.T. Ingolia, J.R. Yates 3rd, and M.W. Hetzer. 2013. Identification of long-lived proteins reveals exceptional stability of essential cellular structures. Cell 154:971-982.

13. Kanemaki, M.T. 2013. Frontiers of protein expression control with conditional degrons. Pflugers Arch. 465:419-425.

14. Faden, F., S. Mielke, D. Lange, and N. Dissmeyer. 2014. Generic tools for conditionally altering protein abundance and phenotypes on demand. Biol. Chem. 395:737-762.

15. Dohmen, R.J., P. Wu, and A. Varshavsky. 1994. Heat-inducible degron: a method for constructing temperature-sensitive mutants. Science 263:12731276.

16. Nishimura, K., T. Fukagawa, H. Takisawa, T. Kakimoto, and M. Kanemaki. 2009. An auxinbased degron system for the rapid depletion of proteins in nonplant cells. Nat. Methods 6:917-922.

17. Holland, A.J., D. Fachinetti, J.S. Han, and D.W. Cleveland. 2012. Inducible, reversible system for the rapid and complete degradation of proteins in mammalian cells. Proc Natl Acad Sci U S A. 109:E3350-E3357.

18. Banaszynski, L.A., L.C. Chen, L.A. MaynardSmith, A.G. Ooi, and T.J. Wandless. 2006. A rapid, reversible, and tunable method to regulate protein function in living cells using synthetic small molecules. Cell 126:995-1004.

19. Park, A., S.T. Won, M. Pentecost, W. Bartkowski, and B. Lee. 2014. CRISPR/Cas9 allows efficient and complete knock-in of a destabilization domaintagged essential protein in a human cell line, allowing rapid knockdown of protein function. PLoS ONE 9:e95101.

20. Zhou, Q., A. Derti, D. Ruddy, D. Rakiec, I. Kao, M. Lira, V. Gibaja, H. Chan, et al. 2015. A Chemical Genetics Approach for the Functional Assessment of Novel Cancer Genes. Cancer Res. 75:1949-1958.
21. Cho, U., S.M. Zimmerman, L.-c. Chen, E. Owen, J.V. Kim, S.K. Kim, and T.J. Wandless. 2013. Rapid and Tunable Control of Protein Stability in Caenorhabditis elegans Using a Small Molecule. PLoS ONE 8:e72393.

22. Iwamoto, M., T. Bjorklund, C. Lundberg, D. Kirik, and T.J. Wandless. 2010. A general chemical method to regulate protein stability in the mammalian central nervous system. Chem. Biol. 17:981-988.

23. Matthews, D.A., J.T. Bolin, J.M. Burridge, D.J. Filman, K.W. Volz, and J. Kraut. 1985. Dihydrofolate reductase. The stereochemistry of inhibitor selectivity. J. Biol. Chem. 260:392-399.

24. Cong, L., F.A. Ran, D. Cox, S. Lin, R. Barretto, N. Habib, P.D. Hsu, X. Wu, et al. 2013. Multiplex genome engineering using CRISPR/Cas systems. Science 339:819-823.

25. Ran, F.A., P.D. Hsu, J. Wright, V. Agarwala, D.A. Scott, and F. Zhang. 2013. Genome engineering using the CRISPR-Cas9 system. Nat. Protoc. 8:2281-2308.

26. Bassett, A.R., C. Tibbit, C.P. Ponting, and J.L. Liu. 2014. Mutagenesis and homologous recombination in Drosophila cell lines using CRISPR/Cas9. Biol Open. 3:42-49.

27. Adams, J.A., C.A. Fierke, and S.J. Benkovic. 1991. The Function of Amino Acid Residues Contacting the Nicotinamide Ring of NADPH in Dihydrofolate Reductase from Escherichia coli. Biochemistry 30:11046-11054.

28. Balciunas, D., K.J. Wangensteen, A. Wilber, J. Bell, A. Geurts, S. Sivasubbu, X. Wang, P.B. Hackett, et al. 2006. Harnessing a high cargocapacity transposon for genetic applications in vertebrates. PLoS Genet. 2:e169.

29. Carette, J.E., M. Raaben, A.C. Wong, A.S. Herbert, G. Obernosterer, N. Mulherkar, A.I. Kuehne, P.J. Kranzusch, et al. 2011. Ebola virus entry requires the cholesterol transporter NiemannPick C1. Nature 477:340-343.

30. Glover-Cutter, K., S. Kim, J. Espinosa, and D.L. Bentley. 2008. RNA polymerase II pauses and associates with pre-mRNA processing factors at both ends of genes. Nat. Struct. Mol. Biol. 15:71-78.

31. Chu, V.T., T. Weber, B. Wefers, W. Wurst, S. Sander, K. Rajewsky, and R. Kuhn. 2015. Increasing the efficiency of homology-directed repair for CRISPR-Cas9-induced precise gene editing in mammalian cells. Nat. Biotechnol. 33:543548.

32. Maruyama, T., S.K. Dougan, M.C. Truttmann, A.M. Bilate, J.R. Ingram, and H.L. Ploegh. 2015 Increasing the efficiency of genome editing with CRISPR-Cas9 by inhibition of nonhomologous end joining. Nat. Biotechnol. 33:538-542.

33. Orlando, S.J., Y. Santiago, R.C. DeKelver, Y. Freyvert, E.A. Boydston, E.A. Moehle, V.M. Choi, S.M. Gopalan, et al. 2010. Zinc-finger nuclease-driven targeted integration into Mamm. Genomes using donors with limited chromosomal homology. Nucleic Acids Res. 38:e152.

Received 04 September 2015; accepted 11 November 2015.

Address correspondence to Department of Biochemistry and Molecular Genetics, University of Colorado School of Medicine, PO Box 6511, Aurora CO.80045. E-mail: david.bentley@ucdenver.edu

To purchase reprints of this article, contact: biotechniques@fosterprinting.com 